\title{
Condiciones estomatológicas en pacientes con cáncer durante y posterior al tratamiento antineoplásico: revisión narrativa de la literatura
}

\author{
Martha Leonor Rebolledo-Cobos*, MsC. ${ }_{1}$, Olga Patrícia Toloza-Gutiérrez, Od, \\ Iran David Alonso-Brujes, $\mathrm{Od}_{1}$
}

Universidad Metropolitana de Barranquilla, Barranquilla, Colombia

Recibido: 30 de junio del 2016 Aprobado: 16 de septiembre del 2016

*Autor de correspondencia: Martha Leonor Rebolledo-Cobos. Estomatológa y Cirujana oral. Maestrante en Genética Molecular. Profesor asistente Universidad Metropolitana, Programa de Odontología, Oficina de Investigaciones, calle 76 N.o 42-78, piso 3, Barranquilla, Colombia. Tel.: 3013847044. Correo electrónico: mrebolledo@unimetro.edu.co

Cómo citar este artículo: Rebolledo-Cobos ML, Toloza-Gutiérrez OP, Alonso-Brujes ID. Condiciones estomatológicas en pacientes con cáncer durante y posterior al tratamiento antineoplásico: revisión narrativa de la literatura. Rev Nac Odontol. 2017;13(24):87-99. doi: http://dx.doi.org/10.16925/od.v12i24.1659

Resumen. Introducción: las consecuencias de los tratamientos antineoplásicos, traen consigo implicaciones bucales y empeoramiento de otras lesiones ya prestablecidas al diagnóstico oncológico, indistintamente del órgano afectado por el tumor. Complicaciones tales como las mucositis orales, candidiasis orales, trismo, xerostomía, caries, entre otras lesiones, suelen suceder con frecuencia, y pueden ser transitorias en el curso de la aplicación de terapéuticas para el cáncer, como, por ejemplo, quimioterapia y radioterapia; otras prevalecen posterior a la finalización de estas terapéuticas. Métodos: se realizó una revisión narrativa de la literatura que mostró la unificación de criterios que involucran la descripción de los patrones clínicos de cada una de las complicaciones orales por el tratamiento oncológico, en relación con cánceres de diferentes localizaciones. Se efectuó una búsqueda bibliográfica en idioma inglés y español usando los siguientes descriptores: "lesiones, boca, cáncer, quimioterapia, radioterapia, complicaciones orales, antes y después, lesions, mouth cancer, chemotherapy, radiotherapy, oral complications, after y before, para las base de datos global Pubmed y Scielo. En esta se seleccionaron 100 artículos publicados en un período de 15 años. Resultados: la mayoría de autores consultados coinciden en afirmar que una amplia gama de tumores malignos tienden a mostrar afectación secundaria en lugares como la cavidad oral, en su mayoría por el tratamiento oncológico instaurado, sin distinción de tipo tumoral y localización del mismo. Conclusiones: la cavidad bucal sigue siendo susceptible a la presencia de estos efectos, los cuales se identifican como eventos citotóxicos sistémicos en tejidos de recambio rápido, como lo es la mucosa bucal.

Palabras clave: boca, cáncer, lesiones, quimioterapia, radioterapia. 


\title{
Stomatological conditions in cancer patients during and after antineoplastic treatment: A narrative review of the literature
}

\begin{abstract}
Introduction: The consequences of antineoplastic treatments bring about oral implications and worsening of other lesions established prior to the oncological diagnosis, regardless of the organ affected by the tumor. Complications such as oral mucositis, oral candidiasis, trismus, xerostomia, caries, among other lesions, occur frequently and may be transient in the course of the application of cancer therapies, such as chemotherapy and radiotherapy; others prevail after completion of these therapies. Methods: A narrative review of the literature was performed to show unification of criteria involving description of the clinical patterns of each oral complication due to oncological treatment, in relation to cancers in different locations. A bibliographic search was conducted in English and Spanish using the following descriptors: "lesiones, boca, cáncer, quimioterapia, radioterapia, complicaciones orales, antes y después, lesions, mouth cancer, chemotherapy, radiotherapy, oral complications, before and after," on the global databases Pubmed and Scielo. In the latter, 100 articles published in a period of 15 years were selected. Results: The majority of authors reviewed agree that a wide range of malignant tumors tend to show secondary involvement in places such as the oral cavity, mostly because of the established oncological treatment, regardless of tumor type and location. Conclusions: The oral cavity remains susceptible to the presence of these effects, which are identified as systemic cytotoxic events in fast-changing tissues, such as the buccal mucosa.
\end{abstract}

Keywords: mouth, cancer, lesions, chemotherapy, radiotherapy.

\section{Condições estomatológicas em pacientes com câncer durante e após à terapia antineoplásica: revisão narrativa da literatura}

Resumo. Introdução: as consequências das terapias antineoplásicas trazem consigo implicações bucais e piora de outras lesões já pré-estabelecidas ao diagnóstico oncológico, indistintamente do órgão afetado pelo tumor. Complicações, tais como mucosites orais, candidíases orais, trismo, xerostomia, cáries, entre outras lesões, costumam acontecer com frequência e podem ser transitórias no curso da aplicação de terapias para o câncer, como quimioterapia e radioterapia; outras prevalecem após a finalização dessas terapias. Métodos: realizou-se uma revisão narrativa da literatura que mostrou a unificação de critérios que envolvem a descrição dos padrões clínicos de cada uma das complicações orais causadas pelo tratamento oncológico, em relação a cânceres de diferentes localizações. Foi feita uma pesquisa bibliográfica nos idiomas inglês e espanhol usando os seguintes descritores: "lesiones, boca, cáncer, quimioterapia, radioterapia, complicaciones orales, antes y después, lesions, mouth cancer, chemotherapy, radiotherapy, oral complications, after y before" nas bases de dados globais Pubmed e Scielo. Foram selecionados 100 artigos publicados em um período de 15 anos. Resultados: a maioria dos autores consultados coincidem em afirmar que uma ampla gama de tumores malignos tende a mostrar afetação secundária em lugares como a cavidade oral, em sua maioria pelo tratamento oncológico instaurado, sem distinção de tipo tumoral e sua localização. Conclusões: a cavidade bucal continua sendo suscetível à presença desses efeitos, os quais são identificados como eventos citotóxicos sistêmicos em tecidos de renovação rápida, como é o da mucosa bucal.

Palavras-chave: boca, câncer, lesões, quimioterapia, radioterapia. 


\section{Introducción}

los cánceres en los diferentes órganos y sistemas poseen evoluciones diferentes, según el patrón de crecimiento y la naturaleza del tumor maligno. Las ubicaciones anatómicas afectadas y aquellos lugares que lo circundan, suelen lesionarse de forma primaria y secundaria debido a la misma enfermedad, o bien por el tratamiento establecido para el control del mismo, lo cual se denomina complicación u efecto secundario del tratamiento oncológico [1]. La cavidad bucal no se encuentra exenta de padecerlos, puesto que constituye un órgano blanco de complicaciones de gran importancia a la hora de prevenirlos y tratarlos, más aún, para el propio paciente oncológico la boca representa prioridad, no solo por la masticación, sino también por la la fonación y la deglución, funciones vitales del sistema orofaríngeo necesarias para una óptima supervivencia.

Otros órganos anexos a la boca como el gusto y el olfato pueden igualmente afectarse de forma secundaria, por lo que es importante resaltar que del cáncer y su tratamiento derivan considerables secuelas funcionales y estéticas, como lo es la afección de estos órganos vitales [2]. Las decisiones sobre el tratamiento oncológico suelen ser complejas y se debe equilibrar la eficacia del mismo con la probabilidad de supervivencia máxima, el potencial funcional y la calidad de vida futura [3-5].

El tratamiento intensivo de una enfermedad como el cáncer produce efectos tóxicos inevitables, algunos irreversibles en las células normales, sobre todo en aquellas de recambio rápido como lo son las del epitelio bucal - e incluso la piel- y todo aquello que contenga mucosa [6-9]. La mayoría de investigadores concuerdan afirmando que estos tejidos - como, por ejemplo, la mucosa oral, e inclusive la gastrointestinal-, suelen ser objetivos primarios de la toxicidad relacionada con el tratamiento a causa de su tasa rápida de renovación celular. Por lo tanto, se encuentran variados estudios que evidencian múltiples efectos sintomáticos durante y posterior a los tratamientos oncológicos, que desencadenan en el paciente un estado significativo agudo de incomodidad e incapacidad, como lo son las mucositis bucales (MB) en sus diferentes estadios, trismo, disfagia y xerostomía, entre otras $[3,10-19]$.
La cavidad oral se considera sensible a los efectos tóxicos directos e indirectos de la quimioterapia y de la radiación (quimio-radioterapia - QRT-), ya que los riesgos se incrementan por el resultado de la conjunción de otros factores: la microbiota compleja y diversa, el trauma en los tejidos orales durante la función oral normal, infecciones fúngicas como candidiasis y otras bacterianas, presencia de cuerpos extraños, o prótesis traumáticas, todo esto sumado a la deficiente higiene oral que suelen tener los pacientes afectados por el cáncer, debido a que la condición inmunosupresora y psicológica del individuo enfermo de cáncer no le permite, en la mayoría de los casos, tener como prioridad la salud bucal [12-18].

Adicional a la cabeza y el cuello, las localizaciones anatómicas del cáncer distantes a la boca, se relacionan de una u otra forma con la presencia de lesiones estomatológicas, durante y posterior a la aplicación de QRT (incluyendo cirugía oncológica), puesto que sus efectos sistémicos son acumulativos e inherentes a los diferentes estados de inmunosupresión oncológica por las que suele cursar un paciente [13-20].

Según los anteriores planteamientos, es necesario ejecutar una revisión narrativa de la literatura que permita unificar las conceptos según la fisiopatología de las complicaciones estomatológicas por las que cursan los pacientes con diferentes diagnósticos oncológicos del cuerpo humano, durante y posterior a la QRT, no sin antes destacar los principales mecanismos de acción fisiopatológicos y terapéuticos de la QRT y la cirugía oncológica.

Se efectuó una búsqueda bibliográfica en los idiomas inglés y español, en las base de datos global Pubmed y Scielo, en la que se seleccionaron 100 artículos publicados en un período de 15 años. Las referencias escogidas se refieren a las condiciones estomatológicas por las que cursan los pacientes con cáncer, antes y después del tratamiento, sin importar el tipo de tumor y la localización anatómica, ni características sociodemográficas tales como sexo, edad, procedencia, raza o nivel de escolaridad, entre otros aspectos. Se emplearon los siguientes descriptores: lesiones, boca, cáncer, quimioterapia, radioterapia, complicaciones orales, antes y después; lesions, mouth cancer, chemotherapy, radiotherapy, oral complications, after y before. 


\section{El tratamiento del cáncer y la cavidad oral}

Las terapéuticas establecidas, así como el uso ideal y actual de las mismas para el manejo del cáncer, se basan en protocolos unidisciplinarios y algunos interdisciplinarios encaminados a aumentar la sobrevida del paciente, en los que intervienen profesionales de la salud de todos los órganos y sistemas que median dichas lesiones durante su evolución, entre ellos el odontólogo y/o estomatólogo. Este profesional debe participar preventivamente desde el momento en el que se diagnóstica de forma clínica e histológica, hasta culminar el tratamiento antineoplásico específico [5,21]. Dicha conducta debe tener un enfoque terapéutico y paliativo (antes, durante y después), el cual comprende: la supresión quirúrgica, tratamiento citotóxico con QRT - esto ocasiona la muerte celular generalizada-, la cirugía y la radioterapia, entre otras alternativas de tratamiento [2].

La quimioterapia consiste en la administración de medicamentos que producen citotoxicidad, reducen, destruyen e inhiben el crecimiento y la reproducción de las células malignas y sanas, proceso también denominado "cito-reducción" [22-35]. De esta terapéutica deriva la aparición de complicaciones en tejidos cuya configuración celular es de recambio continuo y permanente, tales como el epitelio o mucosas de recubrimiento [3642]. El tiempo de administración depende del tipo, las dimensiones del tumor, las toxicidades previstas de los fármacos y el tiempo necesario para recuperarse de esas toxicidades [11, 43, 44].

Hurtado et al., en su revisión, mencionan la clasificación de los medicamentos quimioterapéuticos de uso común: 1. Agentes alquilantes, los cuales afectan directamente el ADN, al evitar la reproducción de las células cancerosas; 2 . Antimetabolitos, que interfieren con el crecimiento del ADN y del ARN al sustituir los elementos fundamentales moleculares; 3 . Las antraciclinas - otro de los medicamentos utilizados- interfieren con las enzimas involucradas en la replicación de ADN, en todas las fases del ciclo; 4 . Los inhibidores de la topoisomerasa, que ayudan a separar las hebras de ADN para que se puedan copiar; y 5. Los inhibidores de la mitosis: detienen la mitosis o evitan que las enzimas sinteticen las proteínas necesarias para la reproducción de las células [11].
La radioterapia como alternativa terapéutica simplemente es energía que se transmite mediante ondas o una corriente de partículas. Funciona afectando irreversiblemente los oncogenes en las células afectadas por el tumor, e incluso las sanas. En tejidos tales como piel, tegumentos y mucosas, estos oncogenes controlan la manera en que las células crecen y se dividen, de modo que cuando la radiación los afecta, estas células ya no puede crecer y dividirse descontroladamente [44]. Con el tiempo, las células mueren, favoreciendo el pronóstico de la enfermedad. Esto significa que la radiación destruye las células cancerosas y reduce el tamaño de los tumores. A diferencia de la quimioterapia, la radiación en oncología se emplea como terapia exclusivamente local o loco-regional, dependiendo de las adenopatías próximas y relacionadas al tumor, como tratamiento principal para tumores pequeños; también en combinación de cirugía-radioterapia o QRT, y en casos en los cuales el paciente presenta tumores cancerosos de mayor tamaño. Posterior a la cirugía oncológica, se puede aplicar radioterapia, bien sea sola o con quimioterapia, o bien como un tratamiento adicional (adyuvante) para eliminar remanentes y depósitos muy pequeños de cáncer que no hayan podido resecarse quirúrgicamente $[45,46]$.

Hurtado et al. también describen cómo para los procedimientos de radioterapia se utilizan habitualmente dos métodos terapéuticos en la aplicación de esta radiación: teleterapia y braquiterapia. La teleterapia o la radioterapia externa emplean varios tipos de radiaciones, como son el bajo voltaje (rayos x), supervoltaje (cobalto 60), megavoltaje (acelerador lineal), y el haz de electrones (fuente eléctrica), de los cuales los más empleados para la terapia de los tumores de cabeza y cuello son el cobalto 60 y el acelerador lineal de partículas [11]. La planeación del tratamiento es según las necesidades del paciente, de acuerdo con el estadio del tumor, su localización y la dosis propuesta por el equipo tratante. A fin de minimizar los efectos secundarios -incluso en la boca- y obtener mayor beneficio terapéutico, la dosis total de radiación se fracciona: se reparte en un número determinado de sesiones y días [11].

La radioterapia braquiterapia consiste en introducir en el interior del organismo isótopos radiactivos. Con esta radioterapia interna se logra administrar altas dosis de radiación a cortas 
distancias, de tal forma que llega muy poca dosis a los tejidos sanos circundantes al implante $[11,44]$. La duración del tratamiento dependerá en gran medida del tipo de isótopo que se haya empleado en el implante y de la cantidad de dosis que se quiera administrar en la zona del tumor, pero generalmente oscila entre minutos y días [11].

La cirugía oncológica permite realizar la completa escisión del tumor y los nódulos linfáticos adyacentes y a distancia al tumor, seguido por una examinación completa histológica por estadio, lo cual tiene implicaciones en el pronóstico y la necesidad o no de utilizar radioterapia coadyuvante; la cirugía también puede usarse para tumores radiorresistentes [45]. Los pacientes que fallecen por cáncer, por lo general, mueren a causa de la malignidad primaria o por metástasis en los nódulos linfáticos, aunque suele ser inusual. De acuerdo con la localización y la etapa en que se encuentre el cáncer, es posible usar uno o más procedimientos a fin de extirpar el tumor, así como para ayudar a restaurar la apariencia y las funciones vitales afectadas por el cáncer o el tratamiento antineoplásico instaurado [45].

Cuando el cáncer es detectado a tiempo, la cirugía radical suele ser el factor curativo y definitivo, mostrando buen comportamiento en cuanto a complicaciones generales y bucales se refiere, debido a que suele ser en una sola intervención y el impacto que ejerce en el estado de salud del paciente no es tan agresivo. Asimismo, le aporta mayores probabilidades de supervivencia, reduciendo indirectamente la incapacidad funcional oral [46].

Investigadores como Escobar et al. manifiestan que, a partir del 2003, se comenzaron a publicar en la literatura casos y series de pacientes afectados, principalmente con mieloma múltiple y cáncer de mama, los cuales presentaban osteonecrósis de los maxilares, y como patrón común en la terapia farmacológica el uso de bifosfonatos intravenosos, relacionando directamente estas complicación orales a la terapia medicamentosa quimioterapéutica [50]. De igual forma, autores como Jeong et al. manifiestan la presencia de otra entidad patológica, la sialadenitis crónica, la cual aparece como una de las complicaciones más frecuentes después de la aplicación de yodo radiactivo (RAI) como terapia para el cáncer de la glándula tiroides, debido a que se encuentra próxima a la cavidad oral. El mismo autor obtiene en su estudio resultados tales como la aparición de xerostomía en un 16,4\% de sus pacientes evaluados cinco años después de la última terapia anticáncer. Esto apoya la teoría según la cual los efectos orales de la radiación se comportan de forma crónica y acumulativa [51].

Autores como Rocha Buelvas et al. manifiestan que el efecto de los agentes quimioterapéuticos sobre los tejidos óseos produce mielosupresión, es decir, comprometen la actividad de fagocitosis celular, reduciendo la producción de anticuerpos por linfocitos B, así como inhibe el desarrollo de los linfocitos $\mathrm{T}$, bloqueando la reacción inflamatoria; también se le responsabiliza por la aparición de lesiones inflamatorias sintomáticas en boca como las MB [52]. Por otro lado, los mismos autores afirman que, directamente, el efecto de la quimioterapia sobre los tejidos periodontales son la razón por la cual el manejo dental y, sobretodo el periodontal, es indispensable, puesto que un epitelio delgado es susceptible a la entrada de bacterias por infecciones sistémicas. Además, los daños en las glándulas salivares causan xerostomía, lo cual afecta aún más las condiciones de inmunidad periodontal o protectoras de la boca, esto sin contar con la suma de mialgias orales o neuropatías dentales [52].

Estos reportes se relacionan con amplias investigaciones que describen la acción de los quimioterapéuticos como incidentes indirectos durante la mielosupresión, y cómo, una vez desaparecen transitoriamente los signos de inflamación por la leucopenia, aparece neutropenia, trombocitopenia y anemia, aumentando la susceptibilidad a infecciones, sobre todo oportunistas como la candidiasis oral, abscesos y otros efectos como el sangrado periodontal [52, 53].

Un sinnúmero de publicaciones e investigaciones manifiestan que los efectos negativos en cavidad oral, a consecuencia de los tratamientos para el cáncer, no distinguen el tipo de órgano responsable de la enfermedad oncológica. Más aún, la relación demuestra cómo al administrar quimioterapia únicamente - puesto que las lesiones orales son imperativas, a diferencia de la radioterapia cuando es aplicada en un radio lejano a la cabeza y el cuello-, no necesariamente aparecen complicaciones estomatológicas directas. Estos autores consultados especifican las numerosas lesiones estomatológicas de pacientes con variables tipos de cáncer de acuerdo con el tratamiento oncológico específico instaurado (véase la tabla 1) [47,49,50]. 
Tabla 1. Relación de lesiones estomatológicas con el tratamiento y diagnóstico oncológico

\begin{tabular}{|c|c|c|c|}
\hline Autores & $\begin{array}{l}\text { Tratamiento oncológico } \\
\text { en orden de importancia }\end{array}$ & $\begin{array}{l}\text { Diagnóstico } \\
\text { oncológico }\end{array}$ & Lesiones estomatológicas relacionadas \\
\hline $\begin{array}{l}\text { Jacobo M, Berkey FJ, } \\
\text { Jones DL, Escobar EA. }\end{array}$ & $\begin{array}{l}\text { - Quimioterapia } \\
\text { - Radioterapia } \\
\text { - QRT }\end{array}$ & Cáncer gastrointestinal. & $\begin{array}{l}\text { MB, sialadenitis, xerostomía, trismo, gloso- } \\
\text { dinia, caries, candidiasis e hipersensibilidad } \\
\text { dental }[47-50] \text {. }\end{array}$ \\
\hline $\begin{array}{l}\text { Young S, Rocha A, Lara- } \\
\text { more GE, Marques MA, } \\
\text { Raber-Durlacher JE, Sonis } \\
\text { ST, Martín M. }\end{array}$ & $\begin{array}{l}\text { - Quimioterapia } \\
\text { - Bifosfonatos }\end{array}$ & Leucemias y linfomas. & $\begin{array}{l}\text { Caries, gingivoestomatitis, MB, sialadenitis, } \\
\text { xerostomía, trismo, glosodinia, osteorradio- } \\
\text { necrósis [51-57]. }\end{array}$ \\
\hline $\begin{array}{l}\text { Deng H, Legañoa A, Mar- } \\
\text { tín M.Hurtado DC. }\end{array}$ & - QRT y cirugía oncológica. & Cáncer de cabeza y cuello. & $\begin{array}{l}\text { MB, xerostomía, candidiasis, trismo, gloso- } \\
\text { dinia, caries, herpes labial, candidiasis oral, } \\
\text { gingivoestomatitis, osteorradionecrósis, glo- } \\
\text { sodinia, glosopirosis, disgeusia }[3,17,57] \text {. }\end{array}$ \\
\hline $\begin{array}{l}\text { Arraztoa J, Martín M. } \\
\text { Hurtado DC. }\end{array}$ & $\begin{array}{l}\text { - } \text { Quimioterapia } \\
\text { - Radioterapia } \\
\text { - QRT } \\
\text { - Cirugía oncológica }\end{array}$ & Cáncer de tiroides. & $\begin{array}{l}\text { MB, xerostomía, hiposialia, trismo, } \\
\text { glosodinia, caries, herpes oral, candidiasis, } \\
\text { estomatodinia }[45,57] \text {. }\end{array}$ \\
\hline González R. & $\begin{array}{l}\text { - } \text { Quimioterapia } \\
\text { - Radioterapia } \\
\text { - QRT } \\
\text { - Cirugía oncológica }\end{array}$ & Cáncer de cuello uterino. & $\begin{array}{l}\text { Gingivoestomatitis, candidiasis oral, herpes } \\
\text { labial [46]. }\end{array}$ \\
\hline Arraztoa J. & $\begin{array}{l}\text { - Quimioterapia } \\
\text { - Bifosfonatos } \\
\text { - Radioterapia } \\
\text { - Cirugía oncológica }\end{array}$ & Cáncer de mama. & $\begin{array}{l}\text { Gingivoestomatitis, candidiasis, herpes } \\
\text { labial y zoster, osteorradionecrósis [45]. }\end{array}$ \\
\hline Arraztoa J, González R. & $\begin{array}{l}\text { - Quimioterapia } \\
\text { - Radioterapia } \\
\text { - Cirugía oncológica } \\
\end{array}$ & Cáncer de próstata. & $\begin{array}{l}\text { Glosodina, gingivoestomatitis, candidiasis, } \\
\text { herpes labial y zoster }[45,46] \text {. }\end{array}$ \\
\hline Deng H. & $\begin{array}{l}\text { - Quimioterapia } \\
\text { - Radioterapia } \\
\end{array}$ & Cáncer de piel y anexiales & $\begin{array}{l}\text { Xerostomía, trismo, glosodinia, herpes, } \\
\text { candidiasis, MB en variables estadios [3]. }\end{array}$ \\
\hline Arraztoa J, Legañoa A. & $\begin{array}{l}\text { - Radioterapia Quimioterapia } \\
\text { - Cirugía oncológica } \\
\text { - Bifosfonatos }\end{array}$ & $\begin{array}{l}\text { Otros (cáncer en musculo, } \\
\text { huesos y glándulas). }\end{array}$ & $\begin{array}{l}\text { Trismo, xerostomía, osteorradionecrósis, } \\
\text { candidiasis, caries por RQT }[45,17] .\end{array}$ \\
\hline
\end{tabular}

Fuente: elaboración propia

\section{Lesiones estomatológicas asociadas al tratamiento del cáncer}

Las lesiones orales que se manifiestan como consecuencia al cáncer y su tratamiento de QRT son comúnmente: $\mathrm{MB}$ en sus diferentes estadios de aparición y gravedad, osteorradionecrósis de los maxilares, trismo, candidiasis oral, sialadenitis, xerostomía, hiposialia, periodontitis agudas, caries-posradiación, necrosis de los tejidos, otros síntomas tempranos como la estomatodinia, disfagia, disfonía, glosodinia y glosopirosis, entre otras, las cuales se describen a continuación, enfatizando la fase de aparición según sea durante y/o después al tratamiento oncológico [36, 47-50].
MB

Reacción inflamatoria auto-limitante muy común a los fármacos de la quimioterapia, o a la RT hiperfraccionada. Se manifiesta característicamente como edema, eritema o ulceraciones dolorosas en mucosas orales, y puede verse exacerbada por factores locales y generales, tales como trauma y presencia de cuerpos extraños (aparatología ortodóntica y/o protésica). Su frecuencia de presentación oscila entre el $70 \%$ y el $80 \%$, y es más común en pacientes jóvenes en quienes los períodos de cicatrización son más cortos. Se clasifica clínicamente y en coincidencia con diversos autores, en grados evolutivos [23, 58-56]: 
- 0. Normalidad

- I. Eritema generalizada: mucosa rosada no dolorosa y con abundante saliva. La fonación suele ser normal.

- II. Eritema: úlceras poco extensas, sintomáticas, se mantiene la deglución de sólidos.

- III. Úlceras extensas, encías edematosas, sintomáticas, saliva espesa; se mantiene la capacidad de deglutir líquidos, disfonía y estomatodinia.

- IV. Úlceras muy extensas, encías sangrantes, infecciones, hiposialia, odinofagia. Sintomatología aguda.

La frecuencia de aparición de estas lesiones se reportan durante y al final de los ciclos pre-escritos de quimioterapia, y al finalizar las últimas sesiones de radioterapia [23, 58-68].

\section{Trismo}

Corresponde a una fibrosis y contracción de los músculos de la masticación, además de daños degenerativos en la articulación temporomandibu$\operatorname{lar}(\mathrm{ATM})$; se inicia de manera gradual a los nueve meses, aproximadamente, luego de haber culminado la radioterapia, manifestándose con una limitación de la apertura de la boca con una distancia inter-incisal menor a $18-20 \mathrm{~mm}$; tiene una prevalencia del 5 al $38 \%$ en los pacientes con cánceres de cabeza y cuello que reciben radioterapia. Afecta la calidad de vida del paciente, debido a que presenta dificultad para hablar, compromiso de la higiene bucal y desnutrición provocada por la dificultad al masticar $[13,69,70]$.

\section{Xerostomía, hiposialia y sialadenitis}

Son lesiones características de pacientes en QRT. La xerostomía es la sensación subjetiva de sequedad bucal debido a la disminución de saliva o hiposialia (una consecuencia de la otra), provocada por una alteración del funcionamiento de las glándulas salivales y/o atrofia de las mismas. Los ácinos glandulares - más que todo de la parótida - se tornan afuncionales, lo cual se traduce en la reducción salival. Los síntomas de xerostomía e hiposialia incluyen sequedad, sensación de ardor o glosopirosis, labios fisurados, queilitis angular, atrofias en la superficie de la lengua, dificultad al usar prótesis y glosodinia. La severidad de la xerostomía depende de la dosis de radiación y del número de glándulas irradiadas; la duración de la misma inicia en el curso de la aplicación de QRT o cualquiera de las mismas de forma individual, y puede prevalecer de forma indefinida e irreversible [71-75]

La sialadenitis corresponde a la inflamación e hipertrofia dolorosa de las glándulas salivales causada generalmente por citomegalovirus (CMv), o por bacterias como el Staphilococus aureus y Streptococus viridans. Esta afección suele ser a consecuencia de la susceptibilidad inmunitaria en glándulas salivales, a la que está expuesto el paciente con cáncer. Se caracteriza por dolor agudo en las glándulas salivales mayores, y dependiendo de la terapia antineoplásica es autolimitante o permanente [76, 77].

\section{Infecciones: candidiasis \\ oral, varicela, herpes}

Este grupo de infecciones fúngicas y virales se desencadenan de forma aguda en condiciones de inmunosupresión, como, por ejemplo, en pacientes sometidos a terapias oncológicas. La irradiación directa afecta las mucosas de la boca, y al obstruir glándulas salivales mayores y menores, da lugar a xerostomía que facilita condiciones habituales para la invasión por el hongo candida, traduciéndose en candidiasis oral. Algunos autores reportan los patrones clínicos de candidiasis pseudomembranosa y atrófica como característicos de los pacientes oncológicos [78]. Por otro lado, las infecciones virales por el grupo de los herpes, manifiestan diferentes enfermedades o condiciones clínicas, las cuales oscilan entre afecciones leves a graves en pacientes que reciben tratamiento del cáncer. La gravedad y el efecto de estas lesiones y secuelas sistémicas se relacionan directamente con el grado de compromiso inmunitario del paciente, el herpes labial o simplemente se reactiva de forma súbita y sintomática, propiciando la aparición de vesículas labiales y perilabiales que tardan un poco más de lo fisiológico en cicatrizar, así como la diseminación en forma de varicela zóster, más agresiva y significativa en términos de gravedad para el paciente. Estas lesiones suelen ser transitorias en el curso del tratamiento oncológico por QRT [49, 78-83]

\section{Osteorradionecrósis de los maxilares}

El hueso, en el área de radiación o próximo a él, se convierte en zona de necrosis hipóxica, 
manifestándose como la denudación del hueso o exposición del mismo al medio oral. Suele presentarse en pacientes con exodóncias, de forma espontánea, por traumas previos o recientes a las terapias para el cáncer, o directamente por el consumo de bifosfonatos. Se manifiesta de forma asintomática durante el curso del tratamiento oncológico o posterior a él, y no posee un pronóstico favorable en la mayoría de los casos. Suele ser irreversible [84-88].

\section{Periodontitis y gingivoestomatitis por QRT}

La periodontitis y gingivitis ulcerosa por QRT aparece en forma de lesiones secundarias, por agravamiento de procesos inflamatorios periodontales preexistentes al establecimiento de terapéuticas. Se manifiesta de manera súbita y aguda en las primeras fases de terapias antineoplásicas, y progresa con abundante sintomatología al sobreinfectarse por la presencia de la microflora patógena preexistente en la boca. Las consecuencias se manifiestan por parte del paciente afectado con movilidad, hasta la pérdida progresiva de sus órganos dentales [89-91].

\section{Caries, abscesos e hipersensibilidad dental}

El riesgo de caries dental aumenta en pacientes sometidos a terapias anticáncer como consecuencia de varios factores, tales como el cambio a una flora cariogénica, la reducción en las concentraciones de proteínas salivales antimicrobianas, o la pérdida de los componentes mineralizantes, sumado a una insuficiente defensa inmunológica por parte del paciente oncológico. Los resultados de esta progresión infecciosa se manifiestan con la presencia de abscesos periodontales de difícil manejo y de grandes sintomatologías, algunos de los cuales suelen producir celulitis u otros estadios complejos de infección oro-fascial. Las lesiones cariosas en los pacientes oncológicos pueden evidenciarse de forma preexistente al cáncer, durante el tratamiento de QRT, o de forma aislada suele exacerbarse. Se evidencia alta destrucción dental y dolor permanente en todos los órganos dentales, lo cual se denomina hipersensibilidad dental. Los micro túbulos dentinales suelen verse expuestos fácilmente al medio oral y favorecer esta sintomatología aguda que puede complicarse a una pulpitis aguda [49, 92-94].

\section{Glosodinia y glosopirosis}

Son síntomas caracterizados por una sensación espontánea anormal descrita por el paciente como quemazón, ardor o escozor, y afecta la mucosa bucal. Los pacientes oncológicos experimentan síntomas tales como alteración del gusto, sensación punzante, xerostomía e intolerancia a las prótesis. Es más característico de la lengua, asociado comúnmente por la presencia de atrofia de las papilas, y se da posterior a la radiación y administración de terapias farmacológicas diversas. Estas lesiones suelen aparecer durante el tratamiento para el cáncer y prevalecer hasta meses, posterior a la última sesión de tratamiento [95-96].

\section{Disgeusia y estomatodinia}

Trastorno en el sentido del gusto que va de la mano con la hiposialia, ya que al carecer de estímulos gustativos la secreción salival refleja residual se ve aún más reducida. El paciente en tratamiento para el cáncer manifiesta característicamente esta afección [97]. Con la exposición de la mucosa oral y faríngea a la RQT, los receptores del gusto se afectan y la sensibilidad del mismo se torna cada vez más comprometida (hipogeusia), o se pierde por completo (ageusia). La etiología se asocia a la neurotoxicidad directa de las células gustativas, xerostomía e infecciones [98-100].

El dolor es un síntoma muy común en los pacientes oncológicos. En la esfera odontológica las causas de dolor pueden ser muy variadas y muy comúnmente co-agregadas por caries, pulpitis, hipersensibilidad dentinaria, prótesis mal adaptadas e infecciones óseas o mucosas. Esta conjunción de entidades sintomáticas se conoce como estomatodinia o dolor oral generalizado, y suele ser transitorio durante la administración de las terapias para cáncer [100].

\section{Discusión}

Estudios realizados por Marques et al. revelan que el 70\% de los pacientes tuvieron pérdida de inserción periodontal durante el período de estudio, siendo esta más frecuente en la mandíbula que en el maxilar. En estos estudios la evaluación se realizó a 27 pacientes con tumores en región de cabeza y cuello, con media de edad de 50 años, antes del 
inicio de la radioterapia y seis a ocho meses al término de la misma. Los pacientes fueron seleccionados con base al tipo de tumor o sitio, dosis de radiación o campo irradiado. Esto evidencia la susceptibilidad de los tejidos periodontales a producir cambios significativos y trascendentales cuando los pacientes son sometidos a terapias antineoplásicas, lo cual coincide con diversas publicaciones revisadas, en las cuales mencionan a las enfermedades periodontales como focos infecciosos exacerbados durante el curso de la enfermedad oncológica [54]. En concordancia con estos argumentos, autores como Hurtado et al. describen en su revisión cómo la cavidad bucal se ve afectada por las radiaciones ionizantes solo cuando se encuentra en el campo o en la proximidad de las zonas donde la radiación se dirige. Por ende, las complicaciones asociadas no solo se aprecian cuando se tratan cánceres propios de la cavidad oral, sino también cuando se irradian lesiones de tiroides, base del tallo cerebral, cerebro $\mathrm{u}$ ojo, entre otros [11].

Otros signos y síntomas destacados en la presente revisión son la dificultad de ejercer acciones como deglutir y masticar. Raber-Durlacher et al. manifiestan la presencia de afecciones prevalentes (tales como la disfagia y la odinofágia) en la mayoría de los estudios que consultaron. Según ellos, estas lesiones se presentaron con mayor frecuencia en pacientes con cáncer de cabeza y cuello, y los problemas de deglución también lo desarrollaban los pacientes con otros tumores malignos de diferentes localizaciones como esófago, faringe, tiroides y tumores de las vías aerodigestivas [55].

Las мв bucales son, sin duda, unos de los efectos y complicaciones más sintomáticos e incapacitantes para los pacientes que cursan por terapias para el manejo del cáncer, y se atribuyen a la acción citotóxica directa que ejercen los agentes quimio-radioterapéuticos. Sonis et al. reportaron un cuadro clínico de cuatro fases que cursa la $\mathrm{MB}$, inicia desde una inflamatoria hasta una cicatrización. Asimismo, aportan que la aparición de estas lesiones comienza a la segunda semana de tratamiento antineoplásico, coincidiendo con diversos autores consultados en esta revisión, quienes afirman que las manifestaciones clínicas iniciales de MB se suelen presentar a la segunda semana de tratamiento y cursan por las cuatro fases evolutivas mencionadas [56-59].

Jacobo, Berkey, Jones, Arraztoa y Legañoa, entre otros autores revisados, concuerdan en sus investigaciones en que las lesiones estomatológicas tales como sialadenitis, xerostomía, trismo, glosodinia, caries, candidiasis oral, hipersensibilidad dental, у мв, son características en los pacientes con cáncer en tratamiento. De igual forma, en que según la cantidad de radiación aplicada, la dosis, la posología, el tipo de quimioterapéutico y los ciclos prescritos de QRT, así como en combinación con cirugía oncológica, determinan la frecuencia y grado de severidad de las complicaciones, algunos de manera aislada o combinados [17, 45, 47-49].

\section{Conclusiones}

Se muestra en la presente revisión narrativa una relación frecuente y directa de afecciones bucales con los tratamientos oncológicos sin distinción de órgano blanco afectado por el cáncer, evidenciando con mayor prevalencia la $\mathrm{MB}$, la xerostomía y el trismo, entre otras.

La búsqueda de literatura mostró evidencia bibliográfica que relaciona directa e indirectamente las complicaciones y/o lesiones orales con las terapéuticas anti-cáncer, así como con diversos tumores malignos de diferentes regiones del cuerpo (mama, próstata, piel, cuello uterino, cáncer gastrointestinal, entre otros tumores distintos a los orales).

La cavidad es un receptor importante de efectos adversos clínicamente significativos como consecuencia de los tratamientos oncológicos. Los efectos adversos que se presentan frecuentemente son la principal queja del paciente oncológico y, muchas veces, el motivo por el que el tratamiento debe suspenderse o modificarse. Por lo tanto, la QRT y la cirugía oncológica, entre otras alternativas terapéuticas, deben planearse teniendo en cuenta dosis, cronograma, ubicación del tumor y estado de salud oral previo del paciente, debido a que la preexistencia de enfermedades bucodentales durante el desarrollo del tratamiento no solo exacerban las condiciones de salud bucal, sino que generan mayores complicaciones locales y sistémicas. Adicionalmente, al conocer las lesiones bucales que se manifiestan durante y después del tratamiento para el cáncer, se hace necesario la implementación de protocolos de atención, los cuales deben ser prioridad para minimizar las consecuencias a largo plazo y proporcionarle al paciente una mejor calidad de vida. 


\section{Agradecimientos}

A Héctor Ruiz-Guerra, Daniela España y Yinni Bermúdez, auxiliares de investigación.

\section{Referencias}

[1] Reyes C, Nambo M, Gallegos F, Silva A, García M, Bustos M. Prevención, tratamiento y rehabilitación oral en el paciente oncológico. Importancia del manejo integral y multidisciplinario. Revista ADM. 2010;67(5):210-16.

[2] Gazel J, Amenábar J. Cuidados odontológicos pre-operatorios adultos mayores con cáncer de boca. Cuidado de adultos mayores con cáncer de boca. Rev Cient Ondontol. 2009;5(2):63-8.

[3] Deng H, Sambrook PJ, Logan RM. The treatment of oral cancer: an overview for dental professionals. Aust Dent J. 2011;56(3):244-52.

[4] Lanza D. Tratamiento odontológico integral del paciente oncológico: Parte I. Odontoestomatol. 2011;13(17):14-25.

[5] González W, Santos A, Carvalho M, Rogério E, Lopes A. Criterios de Evaluación Odontológica Pre-Radioterapia y Necesidad de Tratamiento de las Enfermedades Orales Post-Radioterapia en Cabeza y Cuello. Int. J. Odontostomatol. 2010;4(3):255-66.

[6] Schuurhuis JM, Stokman MA, Roodenburg JL, Reintsema H, Langendijk JA, Vissink A, et al. Efficacy of routine pre-radiation dental screening and dental follow-up in head and neck oncology patients on intermediate and late radiation effects. A retrospective evaluation. Radiother Oncol. 2011;101(3):403-9.

[7] Watters AL, Epstein JB, Agulnik M. Oral complications of targeted cancer therapies: a narrative literature review. Oral Oncol. 2011;47(6):441-8.

[8] Silvestre-Donat FJ, Puente Sandoval A. Efectos adversos del tratamiento del cáncer oral. Av Odontoestomatol. 2008;24(1):111-21.

[9] Reyes X, Sánchez M. Conocimientos y actitudes previas a la quimioterapia en pacientes remitidos a la Liga Colombiana contra el Cáncer. Cuad Latin Admon. 2013;9(16):67-78.

[10] Kędra B, Chomczyk M, Złotkowski M, Stokowska W, Borsuk A, Bicz M, et al. Cytologicalpicture of the oral mucosa in patients with gastric and colon cancer. Folia Histochem Cytobiol. 2012;50(3):375-80.

[11] Hurtado DC, Estrada JH. Oral Complications in Patients Undergoing Radiotherapy: A Review of Literature. Univ Odontol. 2012;31(67):111-29.
[12] Rocha A. Cáncer oral: el papel del odontólogo en la detección temprana y control. Rev Fac Odontol Univ Antioq. 2009;21(1):112-21.

[13] Laramore GE. Role of particle radiotherapy in the management of head and neck cancer. Curr Opin Oncol. 2009;21(3): 224-31

[14] Casariego ZJ. La participación del odontólogo en el control del cáncer oral: Manejo en la prevención, tratamiento y rehabilitación. Revisión Av. Odontoestomatol. 2009;25(5):265-85.

[15] Sabater M, Rodríguez Campillo M, López J, Chimenos E. Manifestaciones orales secundarias al tratamiento oncológico: Pautas de actuación odontológica. Av Odontoestomatol. 2006;22(6):335-42.

[16] Legañoa J, Landrián C, Rodríguez E, Montero A, Agüero A. Evaluación de la atención estomatológica previa a la radioterapia por tumoraciones en cabeza y cuello. AMc. 2010;14(2):1-10.

[17] Legañoa A. Atención estomatológica integral al paciente que recibe radioterapia en cabeza y cuello. AMC. 2013;17(1):10-2.

[18] Cortes J, Ayala C, Cortes J, Cortes R, Salazar L, Castelo $\mathrm{O}$, et al. Oral alterations in children with cancer. Literature review. J Oral Res. 2014;3(4):262-8.

[19] Fernández A, Córdova P, Badenier O, Esguep A. Epidemiological characterization of oral cancer. Literature review. J Oral Res. 2015;4(2):137-45.

[20] Jemal A, Bray F, Center MM, Feriau J. Global cancer statistics. Ca Cancer J Clin. 2011;61(2):69-90.

[21] Salas D, Peiró R. Evidencias sobre la prevención del cáncer. Rev. Esp Sanid Penit. 2013;15(2):66-75.

[22] González C, Bruno G, Salariato O, Álvarez C, Paganini L, Vallejos J, et al. Actualización de la estadificación del cáncer de pulmón. Rev Argent Radiol. 2012;76(4):325-30.

[23] Périé S, Meyers M, Mazzaschi O, De Crouy Chanel $\mathrm{O}$, Baujat B, et al. Epidemiology and anatomy of head and neck cancers. Bull Cancer. 2014;101(5):404-10.

[24] Rebolledo M, Escalante M, Espitia S, Carmona Z. Tipos de tabaquismo como factor de riesgo asociado a cáncer bucal. Reporte de dos casos. Rev Sal UN. 2012;28(3):438-44.

[25] Nachón M, García M, Nachón F, Hernández T, Hernández G. Tabaquismo y cáncer bucal: una revisión teórica. Rev Med Uv. 2010;10(1):30-7.

[26] Groome PA, Rohland SL, Hall SF, Irish J, Mackillop WJ, O'Sullivan B. A population based study of factors associated with early versus late stage oral cavity cancer diagnoses. Oral Oncol. 2011;47(7):642-7.

[27] Szymańska K, Hung RJ, Wünsch-Filho V, Eluf-Neto J, Curado MP, Koifman S, et al. Alcohol and tobacco, and the risk of cancers of the upper aerodigestive 
tract in Latin America: a case-control study. Cancer causes control. 2011;22(7):1037-46.

[28] Pauly JL, O’Connor RJ, Paszkiewicz GM, Cummings KM, Djordjevic MV, Shields PG. Cigarette filter-based assays as proxies for toxicant exposure and smoking behavior-a literature review. Cancer Epidemiol Biomarkers Prev. 2009;18(12):3321-33.

[29] Akbulut N, Oztas B, Kursun S, Evirgen S. Delayed diagnosis of oral squamous cell carcinoma: a case series. J Med Case Reports. 2011;5(291):1-4.

[30] Pelucchi C, Gallus S, Garavello W, Bosetti C, La Vecchia C. Alcohol and tobacco use, and cancer risk for upper aerodigestive tract and liver. Eur J Cancer Prev. 2008;17(4):340-4.

[31] Fernández A, Marshall M, Esquep A. Human Papilloma Virus and oral cancer: Narrative review of the literature. J Oral Res. 2014; 3(3):190-7.

[32] Martín F, Sánchez J, Cano J, Campo J, Del Romero J. Oral Cancer, HPV infection ADN evidence of sexual transmission. Med Oral Patol Oral Cir Bucal. 2013;18(3):439-44.

[33] Picconi MA. Detección de virus papiloma humano en la prevención del cáncer cérvico-uterino. Medicina (B. Aires). 2013;73(6):585-96.

[34] Syrjänen S, Lodi G, von Bültzingslöwen I, Aliko A, Arduino P, Campisi G, et al. Human papilloma viruses in oral carcinoma and oral pontentially malignant disorders: a systematic review. Oral Dis. 2011;17(1):58-72.

[35] Arias SA, Murillo R, Piñeros M, Bravo M, Hernández G, Pardo C, et al. Prioridades de investigación para el control del cáncer en Colombia. Rev Colomb Cancerol 2007;11(3):152-64.

[36] Rodriguez A, Torres D, Robles M, Pachon J, Gonzalez D, Gutierrez J. Cancertreatment-induced oral mucositis: a criticalreview. Int. J. Oral maxillofac. Surg. 2012;41(2):225-38.

[37] Patil VM, Noronha V, Joshi A, Muddu VK, Gulia S, Bhosale B, et al. Induction chemotherapy in technically unresectable locally advanced oral cavity cancers: does it make a difference? Indian J Cancer. 2013;50(1):1-8.

[38] Pavlatos J, Gilliam KK. Oral care protocols for patients undergoing cancer therapy. Gen Dent. 2008;56(5):464-78.

[39] Joshi VK. Review Dental treatment planning and management for the mouth cancer patient. Oral Oncol. 2011;(46):475-9.

[40] Montero A, Hervás A, Morera R, Sancho S, Córdoba S, Corona J, et al. Control de síntomas crónicos: Efectos secundarios del tratamiento con Radioterapia y Quimioterapia. Oncol (Barc). 2005;28(3): 41-50.
[41] Castellanos ME. Las nuevas tecnologías: necesidades y retos en radioterapia en América Latina. Rev Panam Salud Pública. 2006;20(2-3):143-50.

[42] Cano S, Gutiérrez M. Complicaciones de la radioterapia en la cavidad oral. Semergen. 2002; 28(7): 363-9.

[43] Caballero M, Grau JJ, Casellas S, Bernal-Sprekelsen $\mathrm{M}$, Blanch JL. The role of chemotherapy in advanced oral cavity cancer. Acta Otorrinolaringol Esp. 2009; 60(4):260-7.

[44] Martínez B, Cruz M, Echevarría E. Estomatotoxicidad bucal inducida por quimioterapia. Rev Odont Mex. 2014;18(2):89-95.

[45] Arraztoa J. Cirugía oncológica: El porqué de una nueva especialidad quirúrgica derivada de la cirugía general. Rev Chil Cir. 2007;59(1):84-8.

[46] González R. ¿Debemos cuestionarnos el uso de la radioterapia postoperatoria en el carcinoma epidermoide oral? Rev Esp Cirug Oral y Maxilofac. 2012;34(4):143-4.

[47] Jacobo M, Ochoa K. Influencia de una intervención educativa en el conocimiento sobre la prevención de mucositis oral en pacientes sometidos a quimioterapia. Rev Med Hered. 2013;24(4):281-6.

[48] Berkey FJ. Managing the adverse effects of radiation therapy. American Family Physician. 2010;82(4):381-8.

[49] Jones DL, Rankin KV. Management of the oral sequelae of cancer therapy. Tex Dent J. 2012;129(5):461-8.

[50] Escobar EA, López J, Marques MS, Chimenos E. Osteonecrosis de los maxilares asociada a bifosfonatos: revisión sistemática. Av Odontoestomatol. 2007;23(2):91-101.

[51] Young S, Kim HW, Lee SW, Lee BC. Salivary Gland Function 5 Years After Radioactive Iodine Ablation in Patients with Differentiated Thyroid Cancer: Direct Comparison of Pre- and Postablation Scintigraphies and Their Relation to Xerostomia Symptoms. Thyroid. 2013;23(5):609-16.

[52] Rocha A, Jojoa A. Manejo odontológico de las complicaciones orales secundarias al tratamiento oncológico con quimioterapia y radioterapia. CES Odontol. 2011;24(2):71-8.

[53] Laramore GE. Role of particle radiotherapy in the management of head and neck cancer. Curr Opin Oncol. 2009;21(3):224-31.

[54] Marques MA, DibLL. Periodontal changes in patients undergoing radiotherapy. J Periodontol. 2004;75(9): 1178-87.

[55] Raber-Durlacher JE, Brennan MT, Verdonck-de Leeuw IM, Gibson RJ, Eilers JG, Waltimo T, et al. Dysphagia Section, Oral Care Study Group, Multinational Association of Supportive Care in Cancer 
(MASCC)/International Society of Oral Oncology (IsOo), Swallowing dysfunction in cancer patients. Support Care Cancer. 2012;20(3):433-43.

[56] Sonis ST, Lalla RV, Peterson DE. Management of oral mucositis in patients who have cancer. Dent Clin North Am. 2008;52(1):61-77.

[57] Martín M, López M, Cerezo L. Xerostomía postradioterapia. Eficacia de tratamientos tópicos basados en aceite de oliva, betaína y xilitol. Av Odontoestomatol. 2014;30(3):161-70.

[58] Ruiz-Domínguez MR, Tejada-Domínguez FG. Mucositis oral: decisiones sobre el cuidado bucal en pacientes sometidos a radioterapia y quimioterapia conforme a la evidencia. Enferm Global Esp. 2010;9(1):1-22.

[59] Thomson PJ, Greenwood M, Meechan JG. General medicine and surgery for dental practitioners. Part 6 Cancer, radiotherapy and chemotherapy. Br Dent J. 2010;209(2):65-8.

[60] Hurtado DC, Estrada JH. Complicaciones orales en pacientes sometidos a radioterapia: revisión de literatura. Univ Odontol. 2012;31(67):111-29.

[61] Feller L, Essop R, Wood NH, Khammissa RA, Chikte UM, Meyerov R, et al. Chemotherapyand radiotherapy induced oral mucositis: pathobiology, epidemiology and management. SADJ. 2010;65(8):372-4.

[62] Ruiz G, Nervi B, Vargas A, Maíz A. Tratamiento y prevención de la mucositis oral asociada al tratamiento del cáncer. Rev Méd Chile. 2011;139(3): 373-81.

[63] Mora K, Rivero A, Patiño J. Prevalencia de mucositis de cavidad oral de pacientes pediátricos. Centro Javeriano de Oncología. obous Científica. 2009;10(2):7-15.

[64] Molina S, Estupiñán J. Mucositis oral en el paciente con terapia antineoplásica. Medicina UPB. 2010;29(2):135-43.

[65] Peterson DE, Ohrn K, Bowen J, Fliedner M, Lees J, Loprinzi C, et al. Mucositis Study Group of the Multinational Association of Supportive Care in Cancer/International Society of Oral Oncology (MASCC/ISOO).Systematic review of oral cryotherapy for management of oral mucositis caused by cancer therapy. Support Care Cancer. 2013;21(1):327-9.

[66] Schmidt SR, Dias RS, Giordani AJ, Araújo R, Comodo HR. Mucositis in head and neck cancer patients undergoing radiochemotherapy. REEUSP. 2011;45(6):1336-42.

[67] EIilers J, Million R. Clinical update: prevention and management of oral mucositis in patients with cancer. Semin Oncol Nurs. 2011;27(4):1-16.
[68] McGuire DB, Fulton JS, Park J, Brown CG, Correa ME, Eilers J, et al. Systematic review of basic oral care for the management of oral mucositis in cancer patients. Support Care Cancer. 2013;21(11):3165-77.

[69] Bensadoun RJ, Riesenbeck D, Lockhart PB, Elting LS, Spijkervet FK, Brennan, MT. A systematic review of trismus induced by cancer therapies in head and neck cancer patients. Support Care Cancer. 2010;18(8):1033-8.

[70] Chua DT, Lo C, Yuen J, Foo YC. A pilot study of pentoxifylline in the treatment of radiation-induced trismus. Am J Clin Oncol. 2001;24(4):366-9.

[71] Jensen SB, Pedersen AM, Vissink A, Andersen E, Brown CG, Davies AN, et al. Salivary Gland Hypofunction/Xerostomia Section, Oral Care Study Group, Multinational Association of Supportive Care in Cancer (MASCC)/International Society of Oral Oncology (ISOo). A systematic review of salivary gland hypofunction and xerostomia induced by cancer therapies: prevalence, severity and impact on quality of life. Support Care Cancer. 2010;18(8):1039-60.

[72] Sood AJ, Fox NF, O’Connell BP, Lovelace TL, Nguyen SA, Sharma AK, et al. Salivary gland transfer to prevent radiation-induced xerostomia: A systematic review and meta-analysis. Oral Oncol. 2014;50(2):77-83.

[73] García A, Domínguez A, García J, Cancela G, Torres J, Esparza G. Revisión y puesta al día en cáncer de lengua. Av Odontoestomatol. 2013;29(5): 255-69.

[74] Shao ZY, Tang ZS, Yan C, Jiang YT, Ma R, Liu Z, Huang ZW. Effects of intensity-modulated radiotherapy on human oral microflora. J Radiat Res. 2011;52(6):834-9.

[75] Strietzel FP, Lafaurie GI, Mendoza GR, Alajbeg I, Pejda S, Vuletić L, et al. Efficacy and safety of an intraoral electrostimulation device for xerostomia relief: a multicenter, randomized trial. Arthritis Rheum. 2011;63(1):180-90.

[76] Lazos M. Sialadenitis poco frecuentes. Conceptos actuales Rev Med Hosp Gen Mex. 2010;73(2):120-8.

[77] Silberstein EB. Reducing the incidence of 131I-induced sialadenitis: the role of pilocarpine. J Nucl Med. 2008;49(4):546-9.

[78] Lanza D. Tratamiento odontológico integral del paciente oncológico: Parte II. Odontoestomatol. 2013;15(22):46-63.

[79] Martel C, Ferlay J, Franceschi S, Vignat J, Bray F, Forman D, et al. Global burden of cancers attributable to infections in 2008: a review and synthetic analysis. Lancet Oncology. 2012;13(6):607-15.

[80] Kurnatowski P, Moqbil S, Kaczmarczyk D. Signs, symptoms and the prevalence of fungi detected 
from the oral cavity and pharynx of radiotherapy subjects with head and neck tumors, and their susceptibility to chemotherapeutics. Ann Parasitol. 2014;60(3):207-13.

[81] Rueda F, Hernández SE. Prevalencia de Candida albicans aislada de la cavidad oral de pacientes con cáncer. Rev Odontol Latinoam. 2008;0(2):38-41.

[82] Zaldívar G, Martín F, Sosa C, Ávila J, Lloret M, Lara $\mathrm{R}$, et al. Cáncer cérvicouterino y virus del papiloma humano. Rev Chil Obstet Ginecol. 2012; 77(4):31521.

[83] Gligorov J, Bastit L, Gervais H, Henni M, Kahila W, Lepille D, et al. Candidoscope Study Group. Prevalence and treatment management of oropharyngeal candidiasis in cancer patients: Results of the French candidoscope study. Int J Radiat Oncol Biol Phys. 2011;80(2):532-9.

[84] Karagozoglu KH, Dekker HA, Rietveld D, de Bree R, Schulten EA, Kantola S, et al. Proposal for a new staging system for osteoradionecrosis of the mandible. Med Oral Patol Oral Cir Bucal. 2014;19(5): 433-7.

[85] Herrera A, Díaz A, Herrera F, Fang L. Osteorradionecrosis como secuela de la radioterapia. Av Odontoestomatol. 2012;28(4):175-80.

[86] Rui G, Rettore C, De Bona M, Lazaretti N, Crusius M, De Conto F. Fractura Patológica por Osteorradionecrosis de Mandíbula: Relato de Caso. Int J Odontostomatol. 2014;8(1):113-8.

[87] Chrcanovic B, Reher P, Sousa A, Harris M. Osteoradionecrosis of the jaws-a current overview part 1: Physiopathology and risk and predisposing factors. Oral Maxillofac Surg. 2010;14(1):3-16.

[88] Nabil S, Samman N. Incidence and prevention of osteoradionecrosis after dental extraction in irradiated patients: a systematic review. Int J Oral Maxillofac Surg. 2011;40(3):229-43.

[89] Meurman JK, Grönroos L. Oral and dental health care of oral cancer patients: hyposalivation, caries and infections. Oral Oncol. 2010;46(6):464-7.

[90] Cedeño M, Rivas R, Tuliano C. Manifestaciones bucales de los pacientes sometidos a radioterapia en cabeza y cuello, pautas de atención odontológica. Acta Odontol Venez. 2014;52(1):0-0.

[91] Reyes C, Nambo M, Gallegos F, Silva A, García M, Bustos M. Prevención, tratamiento y rehabilitación oral en el paciente oncológico. Importancia del manejo integral y multidisciplinario. Revista ADM. 2010;67(5):210-6.
[92] Hong CH, Napeñas JJ, Hodgson BD, Stokman MA, Mathers-Stauffer V, Elting LS, et al. Dental Disease Section, Oral Care Study Group, Multi-national Association of Supportive Care in Cancer (MASCC)/ International Society of Oral Oncology (ISOo). A systematic review of dental disease in patients undergoing cancer therapy. Support Care Cancer. 2010;18(8):1007-21.

[93] Schuurhuis JM, Stokman MA, Roodenburg JL, Reintsema $\mathrm{H}$, Langendijk JA, Vissink A, et al. Efficacy of routine pre-radiation dental screening and dental follow-up in head and neck oncology patients on intermediate and late radiation effects. A retrospectiveevaluation. Radiother Oncol. 2011;101(3):403-9.

[94] Ros N, Chimenos E, López J, Rodríguez M. Síndrome de ardor bucal: Actualización diagnóstica y terapéutica. Av Odontoestomatol. 2008;24(5):313-21.

[95] Keefe DM, Schubert MM, Elting LS, Sonis ST, Epstein JB, Raber-Durlacher JE, et al. Mucositis Study Section of the Multinational Association of Supportive Care in Cancer and the International Society for Oral Oncology. Updated clinical practice guidelines for the prevention and treatment of mucositis.Cancer. 2007;109(5):820-31.

[96] Russi EG, Corvò R, Merlotti A, Alterio D, Franco P, Pergolizzi S, et al. Swallowing dysfunction in head and neck cancer patients treated by radiotherapy: review and recommendations of the supportive task group of the Italian Association of Radiation Oncology. Cancer Treat Rev. 2012;38(8):1033-49.

[97] Platteaux N, Dirix P, Dejaeger E, Nuyts S. Dysphagia in head and neck cancer patients treated with chemoradiotherapy. Dysphagia. 2010;25(2):139-52.

[98] Epstein JB, Barasch A. Review Taste disorders in cancer patients: Pathogenesis, and approach to assement and management. Oral Oncol. 2010;(46):7781.

[99] Hovan AJ, Williams PM, Stevenson-Moore P, Wahlin YB, Ohrn KE, Elting LS, et al. Dysgeusia Section, Oral Care Study Group, Multinational Association of Supportive Care in Cancer (MASCC)/International Society of Oral Oncology (Isoo). Support Care Cancer. 2010;18(8):1081-7.

[100] Jellema AP, Slotman BJ, Doornaert P, Leemans CR, Langendijk JA. Impact of radiation-induced xerostomia on quality of life after primary radiotherapy among patients with head and neck cancer. Int $\mathrm{J} \mathrm{Ra}-$ diat Oncol Biol Phys. 2007;69(3):751-60. 